\title{
Effect of Topic-prominent Features of Mandarin Chinese on English Writing
}

\author{
Haiyan Han \\ School of Foreign Languages, University of Ji'nan, Ji’nan, Shandong, China
}

\begin{abstract}
Mandarin Chinese features a subject-verb-object word order and lacks grammatical agreement of any sort. It is basically a head-last language with the modifiers preceding the head word. Other prominent grammatical features include serial verb construction, resultative complement and the double nominative constructions. My paper focuses on the role of topic and subject in Mandarin, drawing on three views on Chinese syntactic structures, namely, SVO approach, topic-comment approach, and topic-prominence approach. A comparison is made among the different views and a conclusion is drawn that topic-prominent approach may better capture the complexities of Chinese syntax, which definitely contributes to English writing.
\end{abstract}

Index Terms-Mandarin Chinese, grammatical features, topic-prominence, English writing

Mandarin Chinese is a branch of the Sino-Tibetan language family. It consists of a large varieties of dialects, some of which are mutually unintelligible. But they share the logogrammic writing system. My paper is focused on the Standardized Chinese (also called Putonghua) which is based on the Beijing dialect of Mandarin. Chinese is an analytic language, which means that the words' function is marked syntactically rather than morphologically. In other words, Chinese possesses no grammatical inflections. Categories such as tense, voice, gender, or number (though there are a few plural markers in pronouns and nouns) are not expressed morphologically. It has serval participles to denote aspect (like zhe 1 indicating the continuous aspect, guo4 indicating past perfective), voice (bei4 construction) and to some extent mood $(a 4, y a 4)$.

Chinese features a subject-verb-object (SVO) word order and lacks grammatical agreement of any sort. It has a rich system of classifiers. It is basically a head-last language meaning that the modifiers precede the head words. Unlike English which is a grammatically driven language, Mandarin is a semantically driven language. Elements which are presumed to be inferable from the immediate context are optionally dropped, especially the objects of transitive verbs. Other prominent grammatical features include serial verb construction, resultative complement and the double nominative construction.

\section{BASIC SyntaCtic Features OF ChINESE}

\section{A. Tenses and Aspects}

Tenses in Chinese are not grammaticalized but indicated by expressions of time ('today', 'yesterday', etc.) or simply embedded in context. But aspectual participles are required such as, zhel (imperfective), lel (perfective) or guo4 (experiential), to mark the temporal flow of action or events (Zhao1968, p. 60). For example,
(1) ni3 chi2 le0 me0
you eat ASP Q
'Have you eaten dinner?'

B. Voice

Chinese verbs do not express voice morphologically. The passive particle beit 'by' is used before the verb to indicate passive voice. For example,

(2) gong1chang3 bei4 shaolhui3 le0

factory BEI burn down ASP

'The factory has been burn down.'

The agent is placed after bei as its object if it is required in the context, as shown below.

(3) gong1chang3 bei4 da4 huo3 shao1-hui3 le0

factory BEI big fire burn down ASP

'The factory has been burn down by the big fire.'

\section{Auxiliary Verbs}

Auxiliary verbs in Mandarin, such as neng2 'can', dei3 'must', ke3neng2 or hui4 'may', take substantive verbs as objects and $b u$ 'no' for a negative. Auxiliary verbs usually express the semantic moods of the following verbs. Auxiliary verbs can occur in successive, for example

(4) ta1 ke3neng2 hui4 lai2 me0 
he/she may may come Q

'Will he/she be able to come?'

\section{Pronouns}

Pronouns mainly consist of personal pronouns, demonstrative pronouns, reflexive pronouns, and possessive pronouns. wo3 'I' refers to the speaker, ni3 'you' to the person addressed, and tal 'he/she/it' to the person pointed, either male or female, human or nonhuman. The plural forms of personal pronouns are formed by suffixing menl, resulting in wo3menO 'we/us', ni3men0 'you (plural)' and talmen0 'they/them'. Mandarin does not distinguish subjective personal pronouns and objective personal pronouns. Demonstrative pronouns are zhe4 'this', na4 'that' and na3 'which'. Reflexive pronouns are formed by attaching zi4ji3 'self' to personal pronouns, such as wo3zi4ji3 'myself'. The addition of possessive maker $d e$ after personal pronouns makes possessive pronouns, such as wo3de0 'my', ni3de0 'your'. Possessive pronouns can be dispensed with altogether if the possessor is obvious to the hearer in the context. In this respect Chinese is different from English. For example,

(5) ni3 dei3 bi4zhe0 yan3jing1 xi3 lian3, ke3shi4 dei3

you must close eye wash face, but must

zhang1zhe0 zui3 shua1 ya2

open mouth brush tooth

'You must wash your face with your eyes closed, but brush your teeth with your mouth open'.

\section{E. Prepositions}

Basically, prepositions precede subordinated substantive, such as:

(6) zai4 mei3guo2

in America

'in America'

(7) cong2 qu4nian2

since last year

'since last year'

Prepositions can also take a postposition, or both. For example:

(8) fang2jian1 li3

room in

'in the room',

(9) zhuo1zi0 shang4

table on

'on the table'.

(10) zai4 zhuolzi0 shang4

on table above

'on the table'

\section{F. Classifier}

Individual nouns are modified with specific classifiers. For example,
(11) yi1 tou2 lv2
one CL donkey
'a donkey'
(12) yi1 tiao2 gou3
one $\mathrm{CL} \quad \mathrm{dog}$
'a dog'
(13) yil zhi1 niao3
one $\quad \mathrm{CL}$ bird
'a bird'
(14) yil pi3 ma3 one CL horse
'a horse'

Note that grammatical classifiers are a matter of words and not of things. For example, if a person is referred to as xian1sheng0 'gentlemen', classifier wei4 is preferred, yilwei4 xian1sheng0, but if as ren2 'person', classifier ge4 is preferred, yilge4 ren2. Some nouns take more than one type of classifiers and there are usually slight differences in meaning. Some nouns have no specific classifiers and take only the general classifier ge4, for example, yi1ge4 wen4ti2 'a question', and yilge4 xiao3tou1 'a thief'. Semantically proper names denote unique individual and require no classifiers.

\section{Is CHINESE A SVO, TOPIC-COMMENT, OR TOPIC-PROMINENT LANGUAGE}


Traditionally, many linguists held that Mandarin Chinese has grammatical relations like subjects and objects which appear in fixed positions. Nonetheless, this traditional view that Chinese has the same grammatical features as Indio-European languages (like English) failed to consider the differences in the two languages: subject is a syntactic and semantic element obligatory in English with the exception of imperatives, while it may be optional in Chinese. The notion of Chinese topic was first put forward in Zhao (1968). He argued that the conception of topic and comment is much more appropriate in Chinese than the conception of actor and action. Since then, the two constructions, namely subject-predicate (SVO) and topic-comment, have been the focus of discussion among many researchers. This approach was strongly supported in LaPolla (2009). LaPolla argued for a discourse-based analysis of grammatical relations in Chinese and noted that in Chinese there has been no grammaticalization of syntactic function such as subject or object (p. 29).

In contrast to the foregoing approaches to Chinese grammatical relations, Li \& Thompson (1975) argued that some languages can be more insightfully described by taking the concepts of topic to be basic, while others by taking the notion of subject as basic due to many structural phenomena. They classified world languages into four types, and two major types are subject-prominent language and topic-prominent language with English and Chinese as their respective representatives. A subject-prominent language is a language in which the grammatical units of subject and predicate are basic to the structure of sentences. A topic-prominent language is one in which the information units of topic and comment are basic to the structure of sentence. The following section is devoted to the the comparison of the three approaches.

\section{A. Subject-verb-object Approach}

Traditional Chinese linguists held that subject-object is a clear actor-actor relation. Subject is the performer of the action while object is the receiver of the action. A verb-object construction is an endocentric construction in which the verb governs the object. For example, helcha2 'drink tea', mai3mi3 'buy rice', and kan4 dian4ying3 'see (a) movie'. Besides nouns and pronouns, verbs and adjectives can function as subjects.

$\begin{array}{cllll}\text { (15) da3 } & \text { shi4 } & \text { teng2, ma4 } & \text { shi4 } & \text { ai4 } \\ \text { spank } & \text { COPULAR } & \text { care scold } & \text { COPULAR } & \text { love }\end{array}$

'To spank is to care, to scold is to love.'

The common types of predicate can be verb, adjective, and even nouns. Nominal predicates usually 'represent a class to which the subject is subsumed or expresses a process or event' (Zhao 1968, p. 90). For example,

(16) ta1 tai4tai0 ying1guo2 ren2.

he wife Britain person

'His wife is a British'.

In this example, taltai4taiO 'his wife', as a member, is subsumed under a class yinglguo2ren2 'British'.

(17) ta 1 gan3mao4 le0

he (have a) cold ASP

'He caught a cold.'

In this example, the nominal predicate gan3mao4le0 expresses a fact or event about his health condition.

The approach of subject-verb-object can offer explanation to complex structures. A complex sentence can thus be treated as a complex of two or more minor sentences. It has to be noted that there is no complementizer (like 'that' in English) in Mandarin Chinese.

The subject can also be a full sentence with subject and predicate.

(18) wo3 ren4wei2 ni3 cuo4 le0

I think you wrong ASP

'I think that you are wrong'.

A full sentence can be seen as a clause predicate. For example,

(19) wu3 ge4 pingg2uo3 san1 ge4 lan4 le0

five CL apple three CL spoil ASP

'Three of the five apples have spoiled'.

The clause in (19) "san1 ge4 lan4 le0" is seen as the predicate of the sentence which takes "wu3 ge4 ping2guo3" as its subject.

It is noticeable that the clause predicates more commonly describe the state or characteristics of the main subject.

(20) ta1 xing4 zi0 man4

he temperament patient

'His is patient'.

The argument that the direction of action in a predicate need go outward from subject to object can not always be supported in actual language production. For example,
(21) jiu3 bu4 neng2 he1, yan1 ke3yi3 chou1 wine not can drink, tobacco may smoke
'(You) cannot drink, but (you) can smoke tobacco.'

In Chinese, the verbs hel'drink' and choul 'smoke' generally have a direction of actions from subject to object. But that outward direction is not obligatorily observed as shown in the preceding example. In other word, an object in 
Mandarin is less grammatically governed by the verb.

\section{B. Topic and Comment}

According to Zhao (1968), the grammatical meaning of subject and predicate in a Chinese sentence is topic and comment, rather than actor and action (p. 69). Chinese has a low proportion of applicability of the actor-action meanings and the conception of topic and comment is more appropriate. Topic is literally the subject matter to talk about and the comment is what the speaker comments on the topic. There does not need to be an actor-action relationship between the subject and object, as long as "there is some general relationship of topic and comment between subject and predicate" (p. 70). For example,

(22) zhe4 ben3 shu1 chu1ban3 le0.

this CL book publish ASP

'This book was published.'

Passive voice is used in the literary translation into English but no passive marker (like bei4 'by') is used in this Chinese example. The use of passive markers would not be appropriate here.

$\mathrm{Xu} \&$ Langendoen (1985) specified the topic structure in Chinese, claiming that "topic structure refers to any grammatical configuration consisting of two part: the topic, which invariably occurs first, and the comment, a clause wich follows the topic and says something about it" (p. 1). The topic tends to have a definite reference and the comment has an indefinite reference, as shown in the following example:

(23) shu1 wo3 mai3 le0

book I buy ASP

'I have bought the book.'

The definiteness of topic can also be marked by pronouns, like zhe4ge4 'this', na4ge4 'that', zhe4xiel 'these', and na4xiel 'those'.

The looseness of subject-predicate relations can be found in ellipsis in Chinese. Verbs and co-referential nouns phrases can often be omitted in context. For example,

(24) -ni3 xi3huan1 kan4 dian4shi4 me0

You like watch TV Q

'Do you like watching TV?'

-xi3huan 1

Like

'Yes, I like watching TV.'

(25) zhe4 jian4 yilfu0 tai4 gui4, suo3yi3 wo3 mei2 mai3

this CL clothes too expensive so I not buy.

'This article of clothes was so expensive so I did not buy it.'

The looseness of subject-predicate relation as shown above would be ungrammatical in English. There are cases in which the relation between subject and predicate is so loose that no specific elements can be considered omitted. Topic-comment approach provides explanation for the unique grammatical structures. The topic-comment relations are often met with in Chinese classics. Following is a line of a poem by Li Bai in Tang Dynasty (as cited in Zhao, 1968, p. 71):

(26) yun2 xiang3 yi1shang0 hua1 xiang3 rong2

cloud think garment flower think face

'Clouds remind one of her garments and flowers remind one of her face.'

What is noticeable in this example is the causative use of the word 'think' and it is seldom used anywhere else.

According to $\mathrm{Xu} \&$ Langendoen (1985), some topic structures are formed by movement of a phrase from the comment into the topic node, while some are base-generated without movement. For example,

(27) shui3guo3 wo3 zui4 xi3huan1 ping2guo3

fruit I most like apple

'As for fruit, i like apples most' (p. 27).

In this based-generated construction of (27), the structure would be ungrammatical no matter where the topic originate within the comment clause.

LaPolla (2009) generalized that the structure of the Chinese clause is based on the pragmatic relations of topical vs. focal material with topical elements preceding the verb and focal elements following the verb. There is no need to posit any grammaticalized notions, like 'subject' or 'object' to explain the structure of the clause in Chinese.

\section{Chinese as a Topic-prominent Language}

The topic-comment approach, an information structure analysis, seems to elegantly explain the majority of Chinese clauses but fails to capture the intricacies or nuances of the structural/semantic relations between verbs and the following noun phrases in comment clause. This theory said a lot about topic but little of the internal structure of comment.

The majority of Chinese linguistics tend to support the third view that both topic and subject as different grammatical notions exist in Chinese (Li \& Thompson, 1976, Huang, 1982, Li 1990, Ning 1993). 
The topic-prominent approach claims that Mandarin does have subject-predicate structures. The word order parallels its English translation. Topics, if present in a structure, can be distinguished from the subject: topics are systematically codified by participles like ne0, a0, ya4; topic does not control reflexivization. However, there is no consensus on the definition of the notions. Shi (2000) defined topic and comment on the basis of its structural position, structural relationship with the verb and its course function:

(28)A topic is an unmarked NP (or its equivalent) that precedes a clause and is related to a position inside the clause; a topic represents an entity that has been mentioned in the previous discourse and is being discussed again in the current sentence, namely, topic is what the current sentence is set up to add new information to. The clause related to to the topic in such as way is the comment(p. 386).

A serious weakness of this claim, however, is that the concept of aboutness cannot explain some of the well-formed topic-comment constructions as (29) (Li \& Thompson, 1976).

(29) zhe4-jian4 shi4qing0 ni3 bu4 neng2 guang1 ma2fan2

this-CL matter you not can only bother

yi2-ge4 ren2

yi-CL person

'(As for) this matter, you can't just bother one person'.

The topic zhe4jian4shi4qing0 'this matter' bears no subject-predicate relation with any position inside the comment.

This type of topic-comment construction is called a dangling topic. Dangling topics are not subcategorized by the verb in the comment and therefore have no relation to any position inside the comment. It can be seen from this case that subject and objet are not the only positions for nominal topics to occupy. The positions for topics include the oblique object of invisible adjunct adverbial PPs (Lv, 1986). Pan \& Hu (2002) contested the problems of Shi (2000) and argued that topics in Chinese can be licensed not only by a syntactic gap or resumptive pronouns, but also by a semantic variable which does not have a explicit corresponding syntactic position (p. 382).

The topic-prominent approach met with much criticism that Li and Thompson's taxonomy confuses two level of linguistic analysis, namely grammatical analysis and the actual division of the sentence. In other words, the approach inconsistently uses subject as a syntactic notion while topic as a pragmatic notion. In response, Her (1991) argued that topic has a grammatical function, in parallel to the syntactic notions of 'subject' and 'object'. Her further proposed the term 'frame' to refer to the semantic or discoursal function encoded by the syntactic topic. Huang (retrieved online) held that there a set of Mandarin verbs that subcategorize for a topic in addition to a subject and suggested that the thematic roles of the subcategorized topics are goals and the subjects agents. Some verbs (like na2shou3 'be good at', zuo4zhu3 'take charge of') are identified which semantically select topic NPs as the arguments. For example,
(30) a. zhong1guo2 cai4 wo3 na2 shou3
China cuisine I take hand
'I am good at Chinese cuisine.'
b. *wo3 na2 shou3 zhong1guo2 cai4
I take hand China cuisine

The contrast between grammatical (a) and ungrammatical (b) shows that the topic-position NPs are semantically restricted by the verbs. This restriction can only be contributed to the lexical items (Huang, online source).

Topic is a significant issue in the research of Chinese linguistics. The typological approach initiated by $\mathrm{Li}$ and Thompson and developed by many other linguists captures the complexities of the grammar of Chinese and parses the relationship between sentence structure and function. It offers several syntactic and semantic generalizations across languages.

\section{PS-RULES OF TP CHINESE}

The basic phrase-structure rules of Chinese can be tentatively presented as follows:

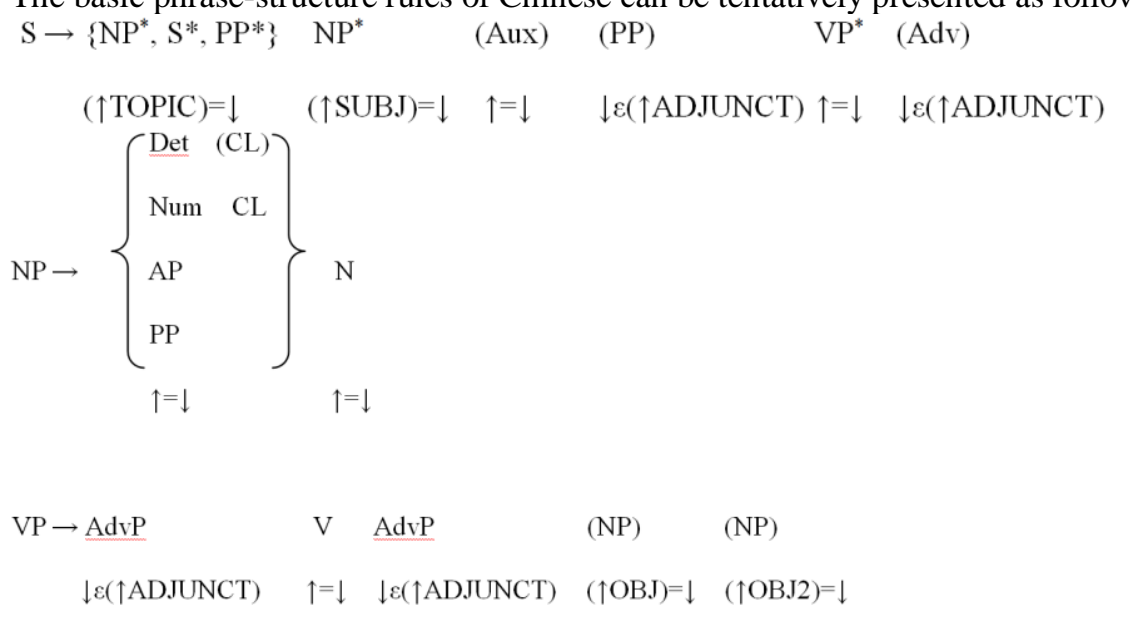




$$
\begin{aligned}
& \mathrm{PP} \rightarrow(\mathrm{P}) \quad \mathrm{NP} \quad \mathrm{P} \\
& \uparrow=\downarrow \quad(\uparrow \mathrm{GF})=\downarrow \quad \uparrow=\downarrow \\
& \mathrm{AP} \rightarrow \text { Adj } \quad \mathrm{N} \\
& \uparrow(\mathrm{ADJUNCT})=\downarrow \quad \uparrow=\downarrow \\
& \mathrm{AdvP} \rightarrow \mathrm{Adv}^{*} \\
& \uparrow=\downarrow
\end{aligned}
$$

Examples are given below to demonstrate the applicability of these rules in Chinese.

(31)

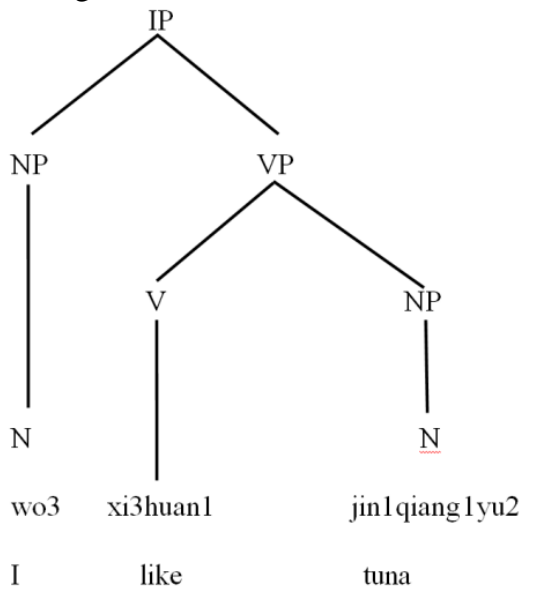

(32)

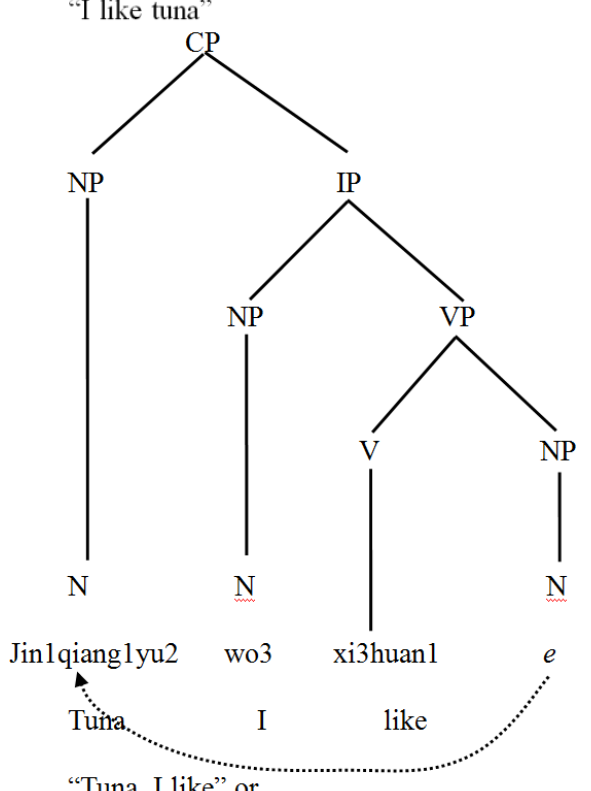

"Tuna, I like" or

"I like tuna" 
(33)

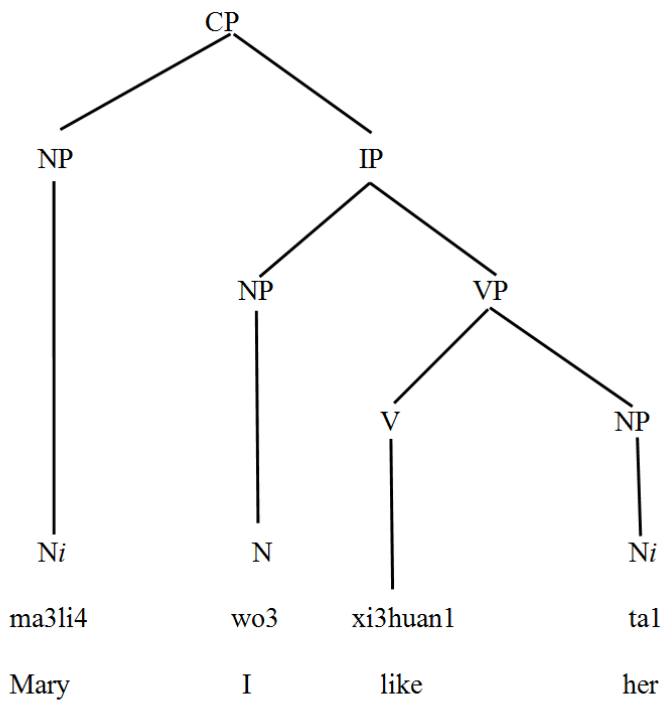

(34)

"I like Mary"

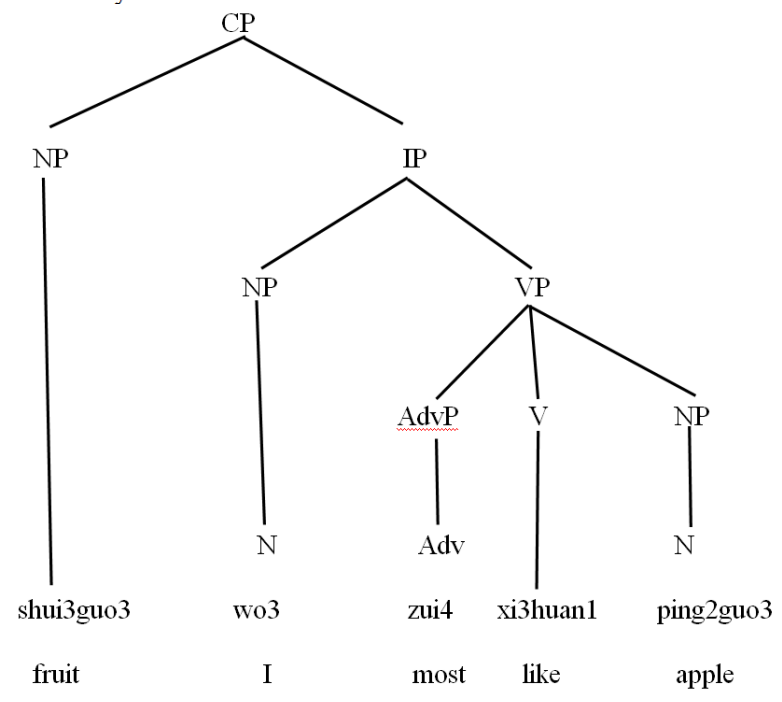

"As for fruit, I like apples most" 
(35)

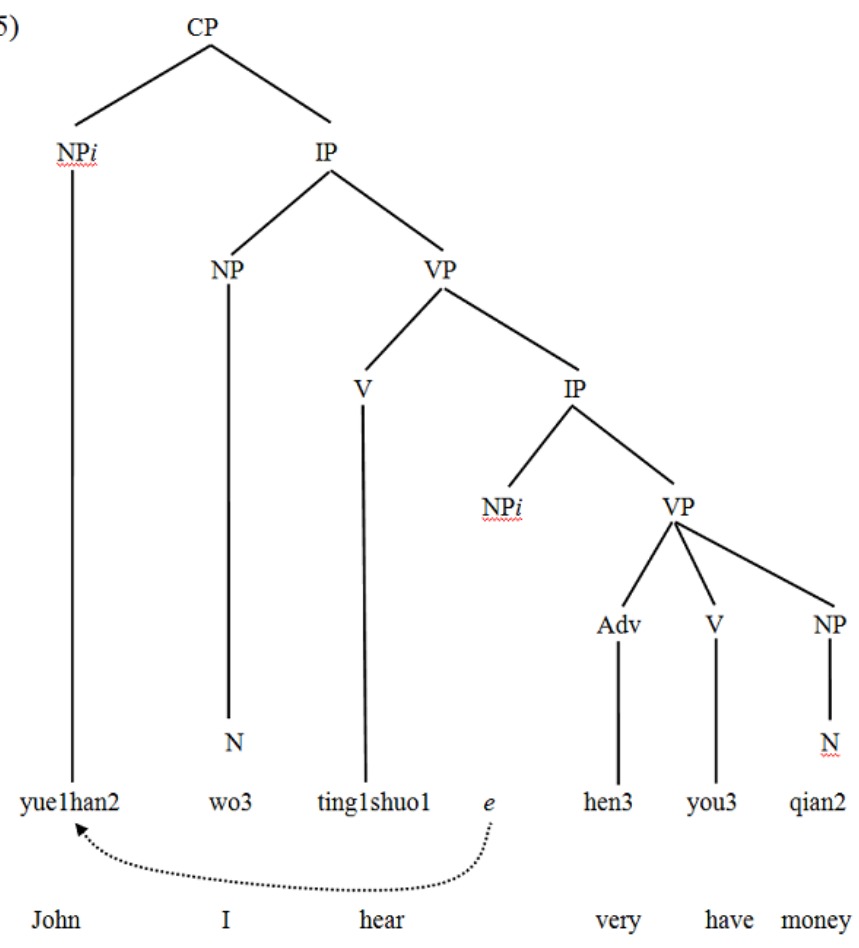

(36)

"I heard that John has a lot of money"

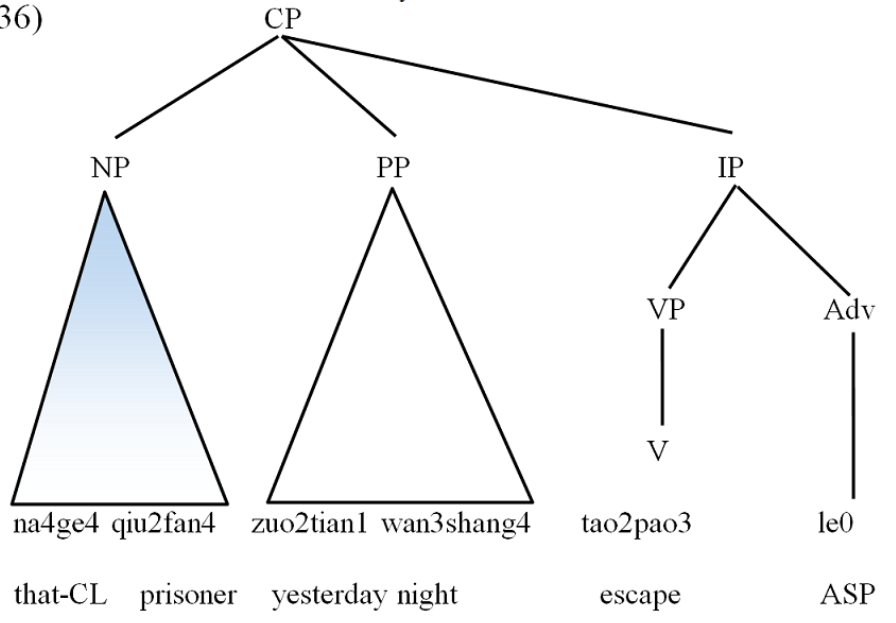

"That prisoner escaped last night"

There is a special topic construction in Chinese called "patient-subject sentence". The property of free argument drop of Chinese is the root of the ambiguity in this construction. For example,

(37) Ji1 chi1 le0

chicken eat ASP

"The chicken has eaten (something)" or

"The chicken has been eaten"

The two readings can be represented in the c-structures as follows: 


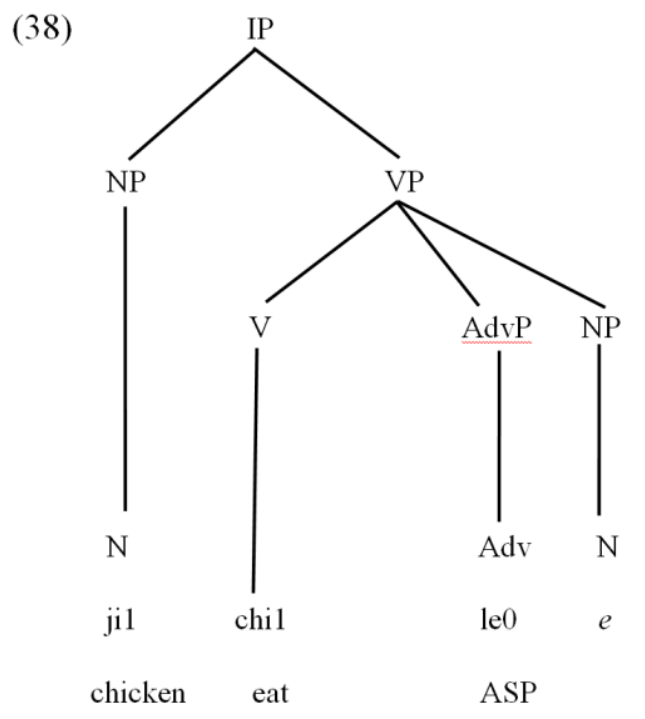

"The chicken has eaten (something)"

(39)

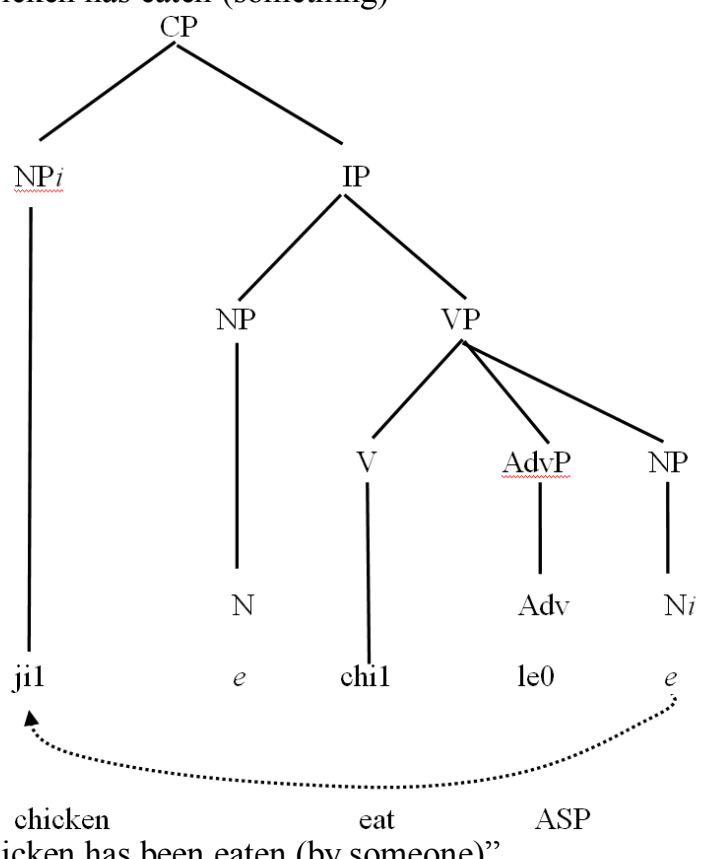

\section{Application of Topic-prominent Features of Mandarin Chinese to English Writing}

As the paper has presented the topic-prominent features of Mandarin Chinese, it has great effect on the English writing. Conventionally, many linguists cling to the belief that Mandarin Chinese has grammatical relations like subjects and objects which emerge in fixed positions. However, this conventional viewpoint that Chinese has the same grammatical features as Indio-European languages (like English) doesn't succeed to think about the distinction in the two languages: subject is a syntactic and semantic element obligatory in English with the exception of imperatives, while it may be selective in Chinese. The conception of Chinese topic was first come up with in Zhao(1968), who argued that the definition of topic and comment is much more appropriate in Chinese than the definition of actor and action. Since then, the two constructions, namely subject-predicate (SVO) and topic-comment, have been the concentration of discussion among many researchers. This approach was strongly advocated in LaPolla (2009), who put forward a discourse-based analysis of grammatical relations in Chinese and noted that in Chinese there has been no grammaticalization of syntactic function such as subject or object (p. 29).

Compared with the foregoing approaches to Chinese grammatical relations, Li \& Thompson (1975) posed that some languages can be more insightfully depicted by taking the concepts of topic to be basic, however others by taking the notion of subject as basic due to many structural phenomena. They classified world languages into four types, and two major types are subject-prominent language and topic-prominent language with English and Chinese as their respective representatives. A subject-prominent language is a language in which the grammatical units of subject and predicate are the basis to the structure of sentences. A topic-prominent language is one in which the information units of topic and comment are basic to the structure of sentence. 
Therefore, since the paper has analyzed the topic-prominent features of Mandarin Chinese, we are supposed to apply it to the English writing. The following are the details: practise your English writing skills at your level. Writing different types of texts, like emails, text messages, essays and letters, is a very important skill for many learners of English, especially those who are learning English for their work or studies.

Choose your level, from beginner to advanced, and start learning today by reading model texts and doing the exercises. Whether you need to improve your English writing skills for work, for studying or to be able to communicate effectively with friends, you'll find practical writing lessons and activities to help you.

Decide how much time you have for your English today and choose a writing activity that you will be able to do from start to finish. When you do the interactive exercises, you can see how well you've done.

To conclude, Mandarin Chinese features a subject-verb-object word order and lacks grammatical agreement of any sort. It is basically a head-last language with the modifiers preceding the head word. Other prominent grammatical features include serial verb construction, resultative complement and the double nominative constructions. My paper focuses on the role of topic and subject in Mandarin, drawing on three views on Chinese syntactic structures, namely, SVO approach, topic-comment approach, and topic-prominence approach. A comparison is made among the different views and a conclusion is drawn that topic-prominent approach may better capture the complexities of Chinese syntax, which definitely contributes to English writing.

\section{APPENDIX. ABBREVIATIONS}

$\begin{array}{ll}\text { ASP } & \text { Aspectual particle } \\ \text { Bei } & \text { Passive marker } \\ \text { CL } & \text { Classifier } \\ \text { De } & \text { Possessive marker } \\ \text { Q } & \text { Interrogative marker }\end{array}$

\section{REFERENCES}

[1] Huang, C. R. Subcategorized topics in Mandarin Chinese. Retrieved on April 10, 2014 from http://cwn.ling.sinica.edu.tw/churen/SubcategorizedTOPICinManda rinChinese.pdf.

[2] Huang, C. T. (1982). Logic relations in Chinese and the theory of grammar. Cambridge, MA: MIT dissertation.

[3] LaPolla, r. J. (2009). Chinese as a topic-comment language. In Studies of Chinese Linguistics: Functional approaches, Xing J (ed.), 9-22. Hong Kong: Hong Kong University Press.

[4] Li, A. (1990). Order and constituency in Mandarin Chinese. Dordrecht: Kluwer.

[5] Li C. N. and Thompson, A. S. (1975). Subject and topic: A new typology of language. In Subject and Topic, Li. C. N. (ed.), 457-490. New York: Academic Press.

[6] Lv, S. X. (1986). The flexibility of Chinese syntax. Chinese Linguistics. 1: 1-9.

[7] Ning, C. (1993). The overt syntax of relativization and topicalization in Chinese. Irvine, CA: University of California, Irvine dissertation.

[8] Pan, H. H \& Hu, J. H. (2002). Representing topic-comment structures in Chinese. Language, Information, and Computation: Proceedings of the $16^{\text {th }}$ Pacific Asia Conference, 382-390. The Korean Society for Language and Information.

[9] Shi, D. X. (2000). Topic and topic-comment constructions in Mandarin Chinese. Language, 76.2: 383-408.

[10] Xu, L. J. \& Langendoen, D. T. (1985). Topic structures in Chinese, Language, 61.1: 1-27.

[11] Zhao, Y. R. (1968). Grammar of Spoken Chinese. Berkeley: University of California.

Haiyan Han was born in Qitaihe, China in 1976. She received her Master's degree in linguistics from University of Jinan, China in 2007.

She is currently an associate professor in the School of Foreign Languages, University of Jinan, Jinan, China. Her research interests include applied linguistics and college English teaching methods. 